\title{
A New Polarization-Reconfigurable Antenna for 5G Wireless Communications
}

Yasir I. A. Al-Yasir ${ }^{1,2}$, Naser Ojaroudi Parchin ${ }^{1}$, Issa Elfergani ${ }^{3}$, Raed A. AbdAlhameed $^{1}$, James M. Noras ${ }^{1}$, Jonathan Rodriguez ${ }^{3}$, Amar Al-jzari ${ }^{2}$ and Waleed I. Hammed $^{2}$

\author{
${ }^{1}$ Bradford University, Bradford BD7 1DP, UK \\ ${ }^{2}$ Basra Oil Training Institute, Basra, Iraq \\ ${ }^{3}$ Instituto de Telecomunicações, Aveiro, Portugal \\ y.i.a.al-yasir@bradford.ac.uk
}

\begin{abstract}
This paper presents a circular polarization reconfigurable antenna for $5 \mathrm{G}$ applications, which is compact in size and has good axial ratio and frequency response. The proposed microstrip antenna is designed on a FR-4 substrate with a relative dielectric constant of 4.3 and has a maximum size of $30 \times 30 \mathrm{~mm}^{2}$ with $50 \Omega$ coaxial probe feeding. This design has two PIN diode switches controlling reconfiguration between right hand circular polarization (RHCP) and left hand circular polarization (LHCP). To achieve reconfigurability, a C-slot rectangular patch antenna with truncated corner techniques is employed by cutting off two corners on the radiating patch. The proposed antenna has been simulated using CST microwave studio software: it has $3.35-3.77 \mathrm{GHz}$ and $3.4-$ $3.72 \mathrm{GHz}$ bands for both states of reconfiguration, and each is suitable for $5 \mathrm{G}$ applications with a good axial ratio of less than $1.8 \mathrm{~dB}$ and good gain of $4.8 \mathrm{~dB}$ for both modes of operation.
\end{abstract}

Keywords: Microstrip Antennas, Reconfigurable, 5G, Circular Polarization.

\section{Introduction}

Many recent books and articles have discussed the use of reconfigurable antennas for "green" flexible RF in 5G applications [1-3]. This topic is of increasing interest for industry because of the requirement for antennas which offer additional functionality and have flexible properties, with the same or smaller physical sizes than previously [4- 6]. The 3.4-3.8 GHz frequency band has been identified as a good candidate for $5 \mathrm{G}$ applications because of the availability of spectrum [7].

Polarization reconfigurable antennas can help to provide protection from interfering signals in variable environments, offering an additional degree of freedom to increase link quality in the form of altered antenna diversity. In addition, they can be used in active read, write tracking and tagging applications and to enhance channel capacity [8]. Several antennas have been developed to deliver reconfigurable polarization characteristics using switches. Su et al. proposed and fabricated polarization re- 
configurable circular-polarized antenna for GPS systems using four photoconductive diodes [9]: by controlling the switching state for each of four diode switches on a microstrip antenna, the radiation polarization can be altered from linear to circular, either left hand or right hand circular polarization (LHCP or RHCP). Khidre et al. [10] designed a single-aperture-fed dual-band reconfigurable antenna for polarization diversity for WLAN applications. The antenna operates at both 2.4 and $5.8 \mathrm{GHz}$ bandwidth using four shorting posts. The impedance bandwidths for the two bands were $3.6 \%$ and $4.3 \%$, respectively, and each band radiates horizontal, vertical and linear polarization, controlled by PIN diode switches. Constant radiation patterns are achieved for different states of polarization with a maximum cross-polarization of -13 $\mathrm{dB}$ and $-9 \mathrm{~dB}$ for the two bands, respectively. Boonying et al. proposed a polarization reconfigurable antenna for WLAN applications at $2.4-2.484 \mathrm{GHz}$ controlled by six PIN diode switches [11]. Other researchers have also presented designs for $5 \mathrm{G}$ applications - for example in [12], an antenna is designed with circular polarization reconfigurability between LHCP and RHCP, applicable in mobile systems.

In this paper, the diversity of a polarization-reconfigurable microstrip antenna with semicircle slot is investigated, based on an antenna with two PIN diode switches giving rise to orthogonal RHCP and LHCP. The orthogonality should occur at least in the direction normal to the antenna, since it is hard to achieve orthogonal polarizations over the whole sphere.

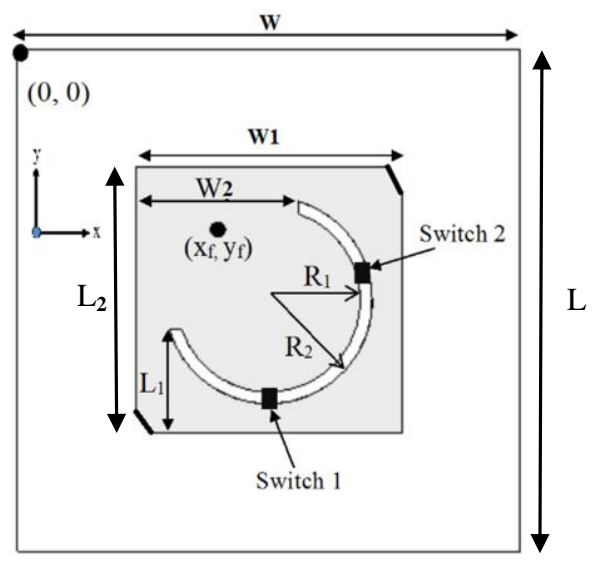

(A) Top view

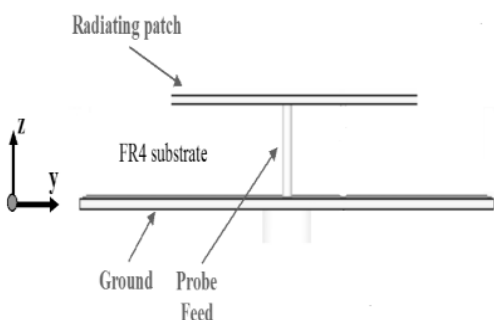

(B) Side view

Fig. 1. Proposed polarization-reconfigurable microstrip antenna

\section{Antenna Design}

In the proposed design, the radiator is fed by a $50 \Omega$ standard probe. An FR-4 substrate is used with $\mathrm{h}=3.2 \mathrm{~mm}, \varepsilon_{r}=4.3$ and loss tangent $=0.02$. The frequency 3.6 $\mathrm{GHz}$ is chosen as the resonance frequency because this frequency is suitable for $5 \mathrm{G}$. A coaxial probe type feed is used. The center of the patch is taken as the origin and the feed point location is given by the co-ordinates $\left(\mathrm{x}_{\mathrm{f}}, \mathrm{y}_{\mathrm{f}}\right)$ from the origin. The loca- 
tion of the feed point is found using CST software, whose parametric optimizer identifies where the input impedance is $50 \Omega$ at the resonant frequency. At $3.6 \mathrm{GHz}$, the optimized feed point location is $\left(\mathrm{x}_{\mathrm{f}}=14.3 \mathrm{~mm}, \mathrm{y}_{\mathrm{f}}=14.6 \mathrm{~mm}\right)$. The antenna is designed with two PIN diodes switches, modeled with a lumped element network which gives $0.9 \Omega$ as the resistance value of a diode in the $\mathrm{ON}$ state and $0.3 \mathrm{pF}$ as its capacitance value in the OFF state. The optimized dimensions are achieved by using the built-in optimizer embedded with the CST software. CST time domain solver has been used with 10 lines per wavelength as mesh density control properties. The dimensions of this antenna are optimized to ensure good matching at resonance. The geometry of the antenna and its optimized dimensions are shown in Fig. 1 and Table 1, respectively.

Table 1. The optimized dimensions of the antenna (Units in $\mathrm{mm}$ )

\begin{tabular}{cccccccc}
\hline $\mathbf{W}$ & $\mathbf{L}$ & $\mathbf{L}_{\mathbf{2}}$ & $\mathbf{W}_{\mathbf{1}}$ & $\mathbf{W}_{\mathbf{2}}$ & $\mathbf{L}_{\mathbf{1}}$ & $\mathbf{R}_{\mathbf{1}}$ & $\mathbf{R}_{\mathbf{2}}$ \\
\hline 30 & 30 & 17 & 17 & 9 & 8 & 6 & 7 \\
\hline
\end{tabular}

\section{Simulation Results}

In this section, the polarization diversity antenna is studied in terms of return loss, radiation pattern, gain and polarization. The simulation results are generated using CST software.

\subsection{Impedance Bandwidth}

The simulation results for the return loss of proposed antenna in Fig. 2 show that, by altering the state of the two PIN diodes, the reflection coefficient $|\mathrm{S} 11|$ is maintained, which is an advantage of this design. At the resonance frequency, the measured effective bandwidths (S11 $<-10 \mathrm{~dB}$ ) for (D1ON, D2OFF) and (D1OFF, D2ON) are $11 \%$ and $8 \%$, respectively, for both states.

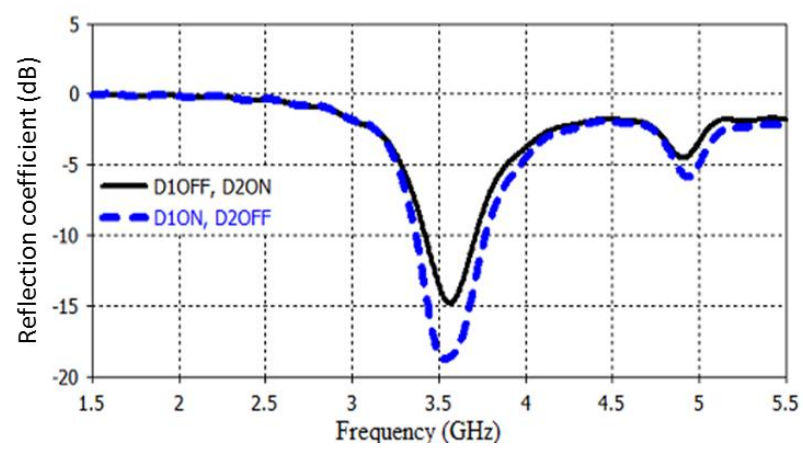

Fig. 2. Return loss for microstrip antenna with two switch modes 


\subsection{Radiation Pattern, Axial Ratios and Gains}

Fig. 3 shows the simulated radiation patterns of the antenna in different switch states (in RHCP and LHCP) at resonance frequencies. Full wave simulation is carried out using CST software. The yz-coordinates are taken into account as the E-plane and xz-coordinates as the $\mathrm{H}$-plane. These results are simulated at $3.6 \mathrm{GHz}$. It is shown that the main lobe direction for (D1On, D2Off of the xz-plane) and (D1Off, D2On of the yz-plane) are on the $\Phi=0$ and $\theta=0$, whereas the main lobe direction for (D1On, D2Off of the yz-plane) and (D1Off, D2On of the xz-plane) are on the $\Phi=10$ and $\theta=-10$, respectively.

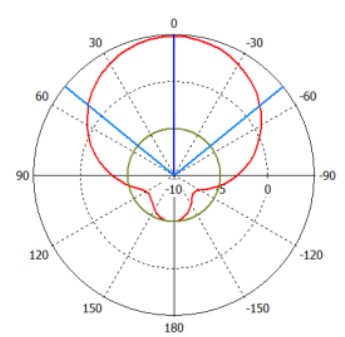

D1On, D2Off xz-plane

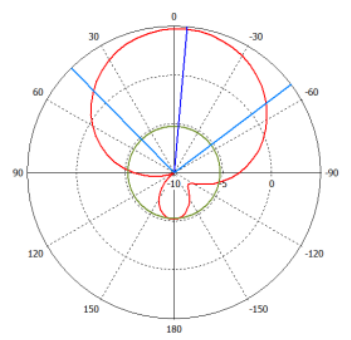

D1Off, D2On xz-plane

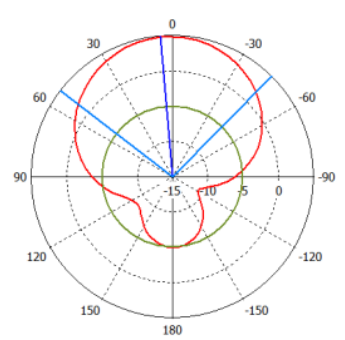

D1On, D2Off yz-plane

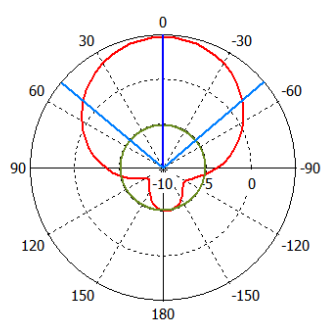

D1Off, D2On yz-plane

Fig. 3. Simulated results for radiation pattern for microstrip antenna

From Fig. 4, the axial ratio can be also observed. At resonance (3.6 GHz), a value of less than $2 \mathrm{~dB}$ axial ratio resulted with the difference between the crosspolarization component and the co-polarization component also less than $2 \mathrm{~dB}$. In simulated results, circular polarization is observed at each state of switching and in the xz-plane and yz-plane. However, the results obtained show circular polarization at broadside and at the most important direction in both xz-plane and yz-plane.

Fig. 5 shows the captured view of an animated field explaining the sense of rotation of circular polarization for the antenna. It shows that the filed distribution of the proposed antenna is rotating in the left-hand circular polarization in the D1Off, D2On state, whereas the field is rotating in the right-hand circular polarization in the D1on, D2off state. 


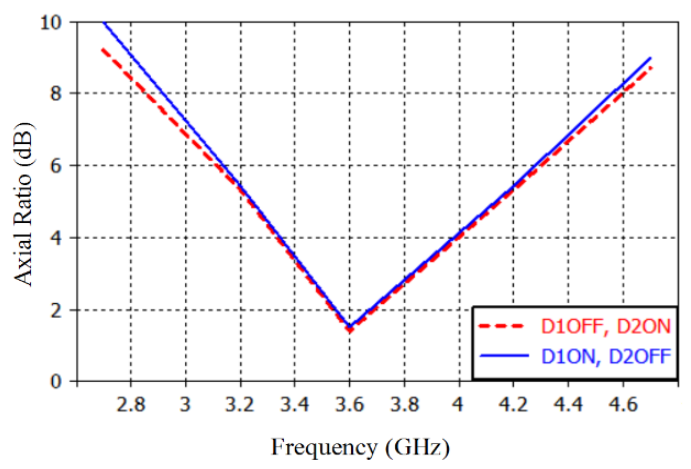

Fig. 4. Simulated axial ratio for the antenna.

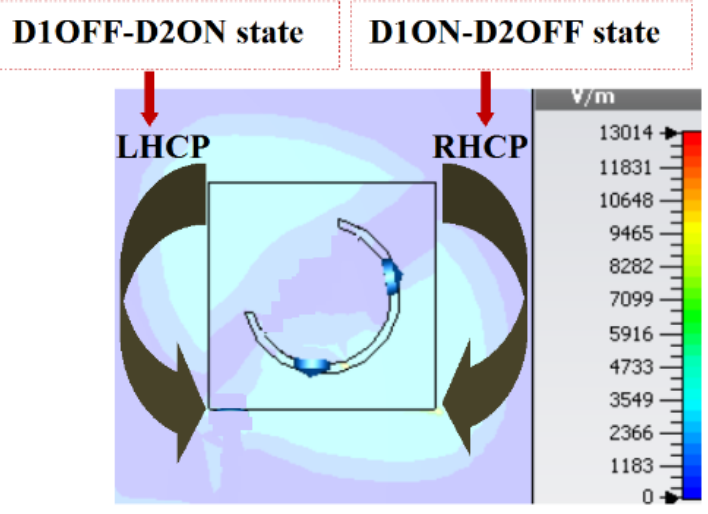

Fig. 5. Captured view of animated field shows the sense of rotation of $\mathrm{CP}$ for the antenna.

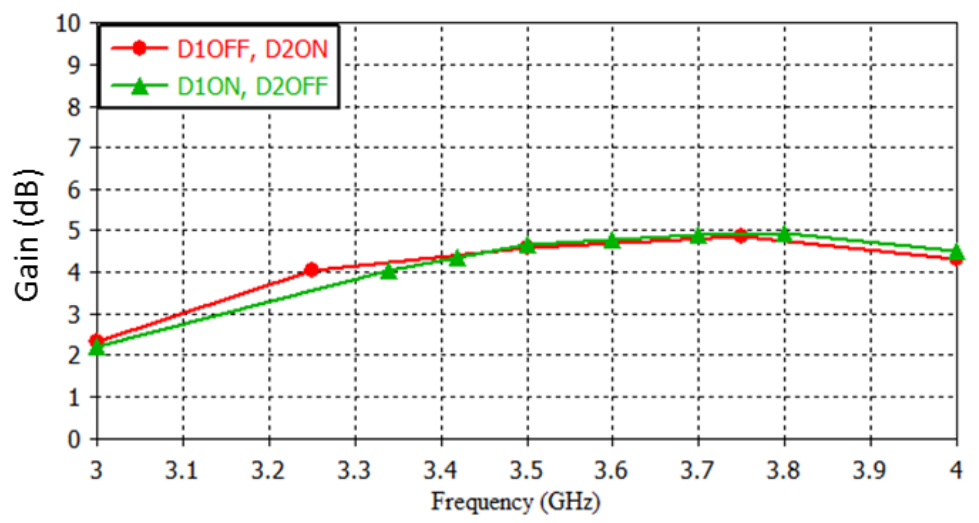

Fig. 6. Simulation results for the realized gain of the proposed antenna 
Fig. 6 shows the simulation results for the maximum realized gain of the proposed antenna. The maximum value for simulated gain is $4.8 \mathrm{~dB}$ for both switching states for the diodes at the resonance frequency.

\section{Comparison with Some Other Existing Structures}

Table 2 compares the proposed polarization-reconfigurable antenna with other antennas with similar configurations and performance. It is noticeable that the proposed antenna is better than others with respect to size, number of switches and also the polarization states obtained compared with the design complexity.

Table 2. Comparison between the proposed structure with some other existing structures.

\begin{tabular}{|c|c|c|c|c|c|}
\hline Ref. & $\begin{array}{c}\text { Antenna size } \\
\left(\mathbf{m m}^{\mathbf{3}}\right)\end{array}$ & $\begin{array}{c}\mathbf{f}_{\mathbf{0}} \\
(\mathbf{G H z})\end{array}$ & $\begin{array}{c}\text { No. of } \\
\text { switches }\end{array}$ & $\begin{array}{c}\text { Design } \\
\text { complexity }\end{array}$ & $\begin{array}{c}\text { Achieved } \\
\text { polarization }\end{array}$ \\
\hline$[9]$ & $70 \times 70 \times 1.6$ & 1.5 & 4 & simple & LP-RHCP-LHCP \\
\hline$[10]$ & $140 \times 80 \times 10$ & 2.4 & 2 & simple & RHCP-LHCP \\
\hline$[11]$ & $80 \times 80 \times 3.2$ & 2.4 & 6 & $\begin{array}{c}\text { very } \\
\text { complex }\end{array}$ & $\begin{array}{c}\text { VP-HP-SP- } \\
\text { RHCP-LHCP }\end{array}$ \\
\hline$[13]$ & $70 \times 70 \times 10.8$ & 2.4 & 2 & simple & RHCP-LHCP \\
\hline$[14]$ & $100 \times 100 \times 3.2$ & 1.5 & 2 & $\begin{array}{c}\text { consists } \\
\text { of two layers }\end{array}$ & HP-VP-RHCP \\
\hline$[15]$ & $67.5 \times 39.3 \times 1.52$ & 2.4 & 2 & simple & LP-CP \\
\hline $\begin{array}{c}\text { this } \\
\text { work }\end{array}$ & $30 \times 30 \times 3.2$ & 3.6 & 2 & simple & RHCP-LHCP \\
\hline
\end{tabular}

\section{Conclusions}

A proposed design for a $5 \mathrm{G}$ circular polarization reconfigurable-microstrip antenna is presented in this paper. The antenna is reconfigurable for circular polarization to cover RHCP and LHCP under the control of PIN diode switches. The proposed design exhibits $11 \%-8 \%$ effective bandwidth with maximum realized gain around 4.8 $\mathrm{dB}$ at $3.6 \mathrm{GHz}$. Only two switches are used for switching the mode of polarization. The antenna characteristics are kept the same at each polarization mode due to symmetry through switching. The antenna covers the $5 \mathrm{G}$ frequency band for potential use in stationary terminals of various wireless applications and it is suitable for WiMax applications and MIMO systems as well.

\section{ACKNOWLEDGMENT}

This work is partially supported by innovation programme under grant agreement H2020-MSCA-ITN-2016 SECRET-722424 and the financial support from the UK Engineering and Physical Sciences Research Council (EPSRC) under grant EP/E022936/1. 


\section{References}

1. Elfergani, I., Hussaini, A., Rodriguez, j. and Abd-Alhameed, R.: Antenna Fundamentals for Legacy Mobile Applications and Beyond. $1^{\text {st }}$ edn. Publisher, Springer (2018).

2. Hussaini, A., Al-Yasir, Y., Voudouris, k., Mohammed, B., Abd-Alhameed, R., Mohammed, H., Elfergani, I., Abdullah, A., Makris, D., Rodriguez, J., Noras, J., Nche, C. and Fonkam, M.: Green Flexible RF for 5G. Fundamentals of 5G Mobile Networks. p. 241272, John Wiley and Sons (2015).

3. Al-Yasir Y., Abdullah, A., Mohammed, H., Mohammedand, B., Abd-Alhameed, R. and Beebe, J.: Design of Radiation Pattern-Reconfigurable $60-\mathrm{GHz}$ Antenna for 5G Applications. Journal of Telecommunications 27(2), 1-6 (2014).

4. Bernhard, J.: Reconfigurable Antennas. Morgan and Claypool Publishers (2007).

5. Al-Yasir, Y., Abdullah, A., Mohammed, H., Abd-Alhameed, R. and Noras, J.: Design of Frequency-reconfigurable Multiband Compact Antenna using two PIN diodes for WLAN/WiMAX Applications. IET Microwaves, Antennas \& Propagation 11(8), 10981105 (2017).

6. Al-Yasir, Y. and Abdullah, A.: Compact Frequency-reconfigurable Antenna for Multiband Wireless Applications. Progress in Electromagnetics Research Symposium, pp. 20362037, Guangzhou, China (2014).

7. Ofcom Homepage, https://www.ofcom.org.uk/

8. Boti, M., Dussopt, L. and Laheurte, J.: Circularly polarized antenna with switchable polarization sense. Electronics Letters 36 (18), 1518-1519 (2000).

9. Su, H., Shoaib, I. and Chen, X.: Optically Tuned Polarisation Reconfigurable Antenna. Proceedings of IEEE Asia-Pacific Conference on Antennas and Propagation, pp. 265-266, Singapore (2012).

10. Khidre, A., Lee, K.-F., Yang, F. and Elsherbeni, A. Z.: Circular Polarization Reconfigurable Wideband E-Shaped Patch Antenna for Wireless Applications. IEEE Transactions on Antennas and Propagation 61(2), 960-964 (2013).

11. Boonying, K., Phongcharoenpanich C. and Kosulvit, S.: Polarization Reconfigurable Suspended Antenna using RF Switches and P-I-N Diodes. The Fourth Joint International Conference on Information and Communication Technology. pp. 1-4, Thailand (2014).

12. Abbas, E. and Mobashsher, A.: Polarization Reconfigurable Antenna for 5G Cellular Networks Operating at Millimeter Waves. Proceeding of 2017 Asia Pacific conference, pp. 772-774., Malaysia (2017).

13. Khaleghi, A. and Kamyab, M.: Reconfigurable Single Port Antenna with Circular Polarization Diversity. IEEE Transactions on Antennas and Propagation 57(2), 555-559 (2009).

14. Nishamol, M., Sarin, V., Tony, D., Aanandan, C., Mohanan, P. and Vasudevan, K.: An Electronically Reconfigurable Microstrip Antenna with Switchable Slots for Polarization Diversity. IEEE Transactions on Antennas and Propagation 59(9), 3424-3427 (2011).

15. Panahi, A., Bao, X. L., Yang, K. O'Conchubhair, O. and Ammann, M. J.: A simple polarization reconfigurable printed monopole antenna. IEEE Transactions on Antennas and Propagation 63(11), 5129-5134, (2015). 